\title{
Development and Performance of the $\mathrm{KTeV}$ Transition Radiation Detector System
}

\author{
Nickolas Solomey ${ }^{\text {a }}$ \\ (For the KTeV Collaboration) \\ - Enrico Fermi Institute, The University of Chicago, USA
}

\begin{abstract}
A large area, nine chamber transition radiation detector system was designed and constructed for the $\mathrm{KTeV}$ rare Kaon decay experiment E799 at Fermilab. The development and performance of this particle identification detector system will be described. The design goal of this system was to be better than 100:1 $e / \pi$ rejection at $90 \%$ electron acceptance; however, the system performed much better.
\end{abstract}

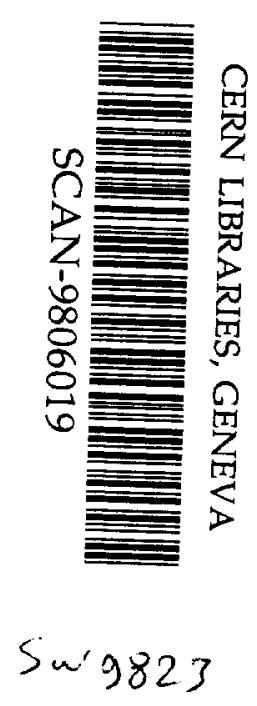

\section{Particle Identification Requirements}

The main physics goal of the $\mathrm{E} 799$ part of the $\mathrm{KTeV}$ experiment is the search for the rare Kaon decay $K_{\text {long }} \rightarrow \pi^{0} e^{+} e^{-}[1]$. Achieving this goal not only required a large number of Kaon decays, but also needs very good particle identification, namely the ability to distinguish electrons from pions. A lot of the particle identification would come from the CsI calorimeter. With better than $1 \%$ energy resolution expected from the CsI calorimeter this put the $e / \pi$ discrimination at better than 500:1 for 90\% electron acceptance. However, more particle identification was needed to be able to achieve the lowest possible branching ratio limit for the main rare Kaon decay search. Simulations indicated that an additional 100:1 $e / \pi$ discrimination at $90 \%$ electron acceptance would put the final achievable goal for the branching ratio at $7 \times 10^{-11}$, close to the theoretically expected region between $10^{-12}$ and $10^{-11}$. More rejection above this would be useful to improve this further, but it would not necessarily translate directly into reducing this limit, due to other limiting factors.

The experiment has the Tevatron proton beam of $800 \mathrm{GeV} / \mathrm{c}$ impacting on a target. A neutral beam is created by collimators and sweeping magnets. The Kaons have a high momentum in the lab and the subsequent decays produce pions or electrons with a large momentum, 20 to $200 \mathrm{GeV} / \mathrm{c}$ for the pions 
and 5 to $50 \mathrm{GeV} / \mathrm{c}$ for electrons. One particle identification method that can work over the whole range from 5 to $200 \mathrm{GeV} / \mathrm{c}$ is transition radiation [2]. So this was a very reasonable choice over other methods such as time of flight, Cherenkov or $\mathrm{dE} / \mathrm{dx}$, since these alternative options did not provide particle identification for the highest momentum tracks.

\section{Detector Design}

The KTeV TRD design is shown schematically in figure 1; it consists of three main parts: the radiator material, entrance window and gaseous detector volume. Each part had to be carefully optimized and designed for maximal performance. Some of these optimizations can be found in a previous publication [3], or in the final design report of the TRD system [4]. Presented here is only a brief description of the most important final parameters of the detector system.

The radiator is a $15 \mathrm{~cm}$ thick mat of randomly oriented $12 \mu \mathrm{m}$ diameter polypropoline $\left(\mathrm{CH}_{2}\right)$ fibers, lightly packed with a density of $0.5 \mathrm{gm} / \mathrm{cm}^{3}$. The gaseous detector needed at least $24 \mathrm{~mm}$ of Xenon gas for good X-ray detection but the charge collection time in such a thick gas volume would have been $1 \mu \mathrm{s}$; however, with a MWPC this time could be cut in half by the two directions of drift while another factor of two in the speed of charge collection came from using two back-to-back MWPC. These are referred to as the front and back readout plane. The detectors are large area, $2.1 \times 2.1 \mathrm{~m}^{2}$, and have the anode wires in the central half of the chamber ganged in groups of two, while the outer half of the chamber has the anode wires ganged in groups of four; this was done only to save expenses by reducing the number of electronics and ADC channels. The gas chosen was $80 \%$ Xenon and $20 \% \mathrm{CO}_{2}$; this had a fast charge collection time of $225 \mathrm{~ns}$ and a decent energy resolution for $\mathrm{X}$-rays, $21.5 \%$ FWHM at $8 \mathrm{keV}$. The gas system was a complete recirculation gas system that only required the full volume to be filled with Xenon once and then occasionally a makeup gas was added to compensate for losses. During the full 14 month run of the experiment this consisted of less than an additional volume of the whole TRD system, a complete description of the recirculating gas system is published elsewhere [5].

The key to a successful TRD system is not just in the optimization of the radiator or gaseous $\mathrm{X}$-ray detector but also depends a lot on the ability to get as many of the TR X-rays from the radiator into the gaseous detector; a good entrance window design was essential. Particular attention was paid to the ability to make a window design that would work on a large area, hence a double window with two aluminized mylar foils $50 \mu \mathrm{m}$ thick with a buffer gas

that had the same density as the Xenon mixture was used. This buffer gas had 
to be extremely transparent to TR X-rays in the range of 5 to $25 \mathrm{keV}$. The final buffer gas was a mixture of $80 \% \mathrm{C}_{2} \mathrm{~F}_{6}$ and $20 \% \mathrm{CO}_{2}$. By making a double window with a heavy buffer gas this assured that the inner window did not move by more than $100 \mu \mathrm{m}$, which allowed the thick $1.5 \mathrm{~mm}$ gas gap between the window and the first plane of cathode wires to be an active part of the gaseous detector volume. The final window design, and each TRD made, was tested for X-ray transparency and efficiency of X-ray detection by the use of a triggered $\mathrm{X}$-ray source, ${ }^{65} \mathrm{Zn}$ or ${ }^{54} \mathrm{Mn}$, which has a high energy gamma ray that is detected in a $\mathrm{NaI}$ crystal, and the daughter nuclei produced has a prompt decay isotropic emitted X-ray, 8.0 and $5.4 \mathrm{keV}$ respectively. The final TRD window design transparency and $\mathrm{X}$-ray detection efficiency of the detector can be seen in figure 2 and is consistent with design expectations.

\section{Performance in the Experiment}

The TRD system consists of nine large area TRDs with radiators. The total amount of material this system had was $12.5 \%$ of a radiation length which was a minimal intrusion of material on the CsI electro-magnetic calorimeter's energy resolution. Each radiator had two holes, $15 \times 15 \mathrm{~cm}^{2}$, for the neutral beam to pass through and the MWPC anode wires were plated to make their wire diameters five times bigger in this hole area. This had the effect to completely deaden the detector in the beam region where there was no radiator material, hence no TR X-rays. This detector system was only installed during the E799 rare Kaon decay part of the experiment. It was operated for six months during the 14 month total fixed target running period of the Tevatron accelerator in 1996 and 1997.

Each TRD had a ${ }^{55} \mathrm{Fe} 5.9 \mathrm{keV} \mathrm{X}$-ray source mounted in the corner of each chamber. The gain of the TRD was constantly monitored and the high voltage regulated to keep the gain constant. This was necessary for two reason. First, since the TRD is a detector that is measuring pulse height to look for X-rays above a minimum ionization signal, any variation in gain would have caused changes in the $e / \pi$ rejection and the performance for particle identification would vary with temperature and pressure variations during the run. So by controlling the gain this gave a reliable constant performance. Secondly, the TRD system also used the pulse height information online to generate a level-2 trigger at reduced rejection to help reduce the various trigger rates. Without gain control this trigger information would be varying, causing changes in trigger conditions which would be unacceptable for precession physics analysis.

The pulse height of the front MWPC plane for one TRD detector in a single run with an electron and pion tagged sample is shown in figure 3 . The pion has an ionization that is mostly at low pulse heights while the sample identified 


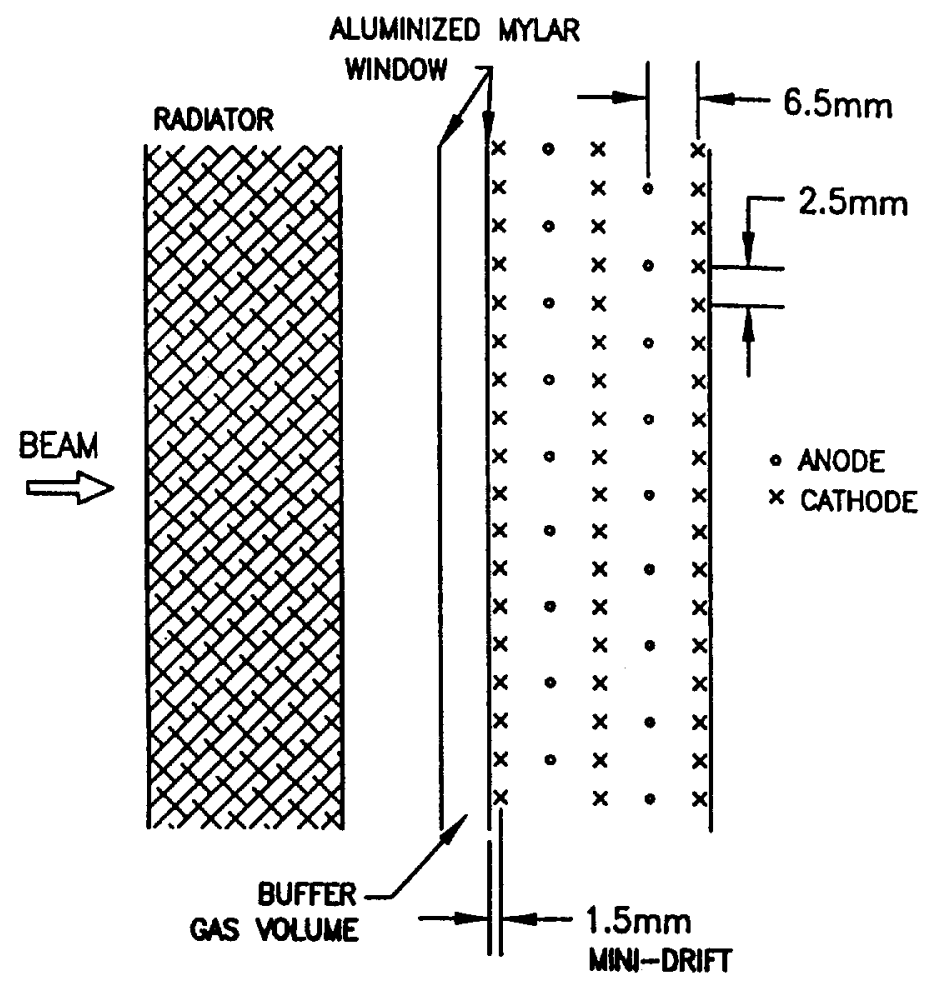

Fig. 1. Schematic layout of the TRD detector. The MWPC uses $25 \mu \mathrm{m}$ diameter wires shown as an 'o' for the anode and $90 \mu \mathrm{m}$ diameter wires shown by an ' $x$ ' for the cathode.

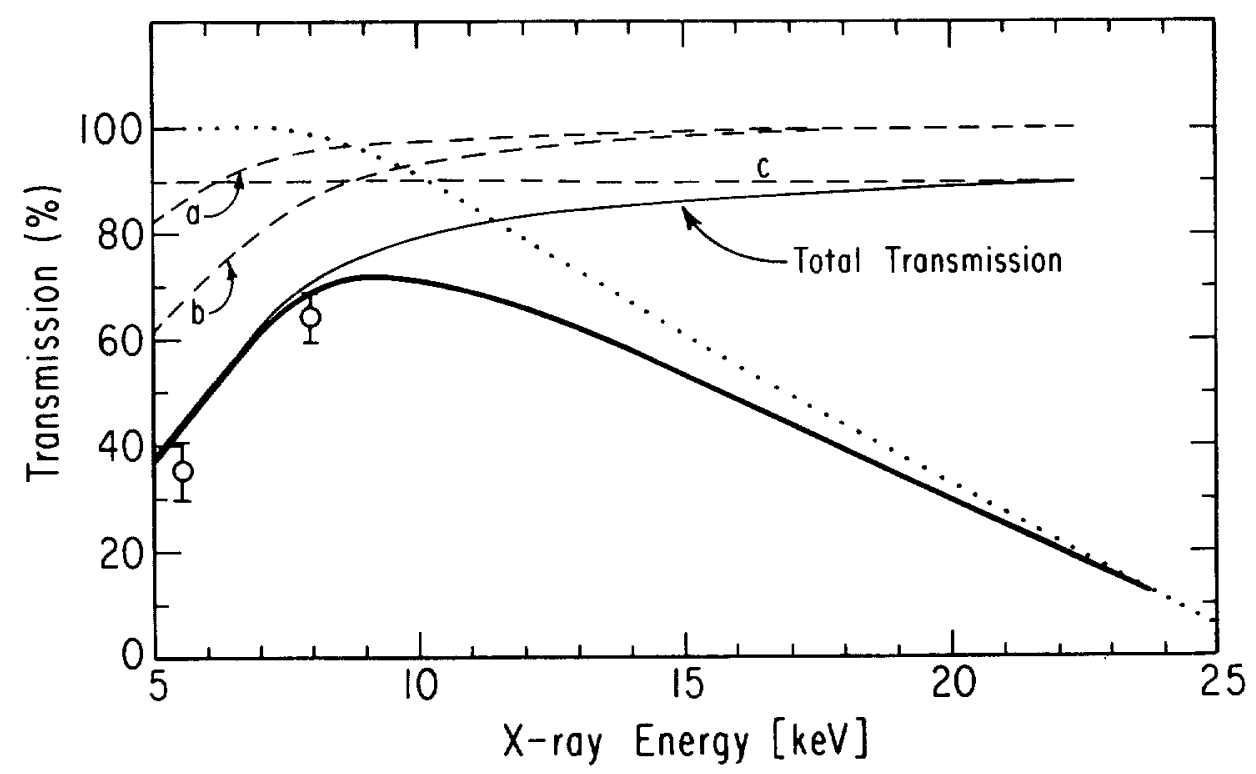

Fig. 2. The X-ray transparency of the many components are shown as dashed lines, the solid line is the final net X-ray transparency for this window design. The X-ray sensitivity in the MWPC is the dotted line, when combined with the net transparency of the window design this yields the bold line for the total X-ray sensitivity. The two points with error bars are from tests of this design. 
as electrons has pulse heights that cover the whole range above the ionization signal, i.e. TR X-rays adding to the ionization signal. A portion of the online event display of a single event is shown in figure 4 . The ionization in the two planes of eight chambers is shown, open squares represent ionization consistent with only an ionization signal, and filled squares pulse heights with ionization that is certainly above minimum ionizing (the difference between open and filled squares for this plot was set at 325 counts in figure 3 ). One track is clearly distinguishable as a pion and the other an electron from the TRD system. This shows how the detector system can perform to identify electrons from pions. Online a similar single threshold method was used as part of the trigger. The actual particle identification in the offline analysis was done by performing a 16 measurement maximum-log-likelihood measurement. This is done by taking the 16 fold product of the ratio of the probability that the pulse height measured in a single plane was from the pion or electron distribution. A distribution of events from an early run is shown in figure 5 , by placing a cut at -5.5 on the log-likelihood this keeps $90 \%$ acceptance for electrons and gives a $e / \pi$ rejection factor of $276: 1$ with an error of \pm 38 . This value seems to be consistent with most of the data taken with the TRD system over its entire operation in the experiment.

\section{Conclusion}

The KTeV TRD system performed reliably over a longer period of time, 14 months, and has a preliminary $e / \pi$ rejection of $276: 1$ for $90 \%$ electron acceptance. This is better than the design goal. Since the TRD is a gaseous detector that performs particle identification by looking at the pulse height of the ionization signal along a track, sources, and a high voltage control program that monitored the gain from the source pulses height were used to assure stability over time. The TRD system in the E799 part of the $\mathrm{KTeV}$ experiment will be a useful detector in the analysis of $K_{\text {long }} \rightarrow \pi^{0} e^{+} e^{-}$as well as $K_{l o n g} \rightarrow \pi^{0} e^{+} e^{-} \gamma$, $K_{\text {long }} \rightarrow \pi^{+} \pi^{-} e^{+} e^{-}$, and $\Xi^{0} \rightarrow \Sigma^{+} e^{-} \overline{\nu_{e}}$.

\section{Acknowledgment}

A detector system of this size and complexity during its many phases (design, construction, operating and analysis) needed a large group effort and I would like to thank my many colleagues who worked with me on making this system such a success: M. Arenton, R. Armstrong, L. Bellantoni, B. Cox, G. Graham, K. Hagan, D. Hicks, B. Hsiung, V. Jejer, K. Kephart, J. Krider, B. Lavoy, S. Ledovskoy, C. McManus, L. Morrison, E. Pod, B. Quinn, E. Ramberg, S. 
Strecker, Y.W. Wah, and E. Zimmerman.

\section{References}

[1] K. Arisaka et al., Report No. FERMILAB-580-1992.

[2] B. Dolgoshein, Nucl. Instr. and Methods, A326 (1993) 434.

[3] G. Graham et al., Nucl. Instr. and Methods, A367 (1995) 225.

[4] G. Graham et al., TRD Design Report, Internal KTeV note No. 185, 2 Feb. 1994.

[5] J. Krider et al., Nucl. Instr. and Methods, A278 (1989) 660. 


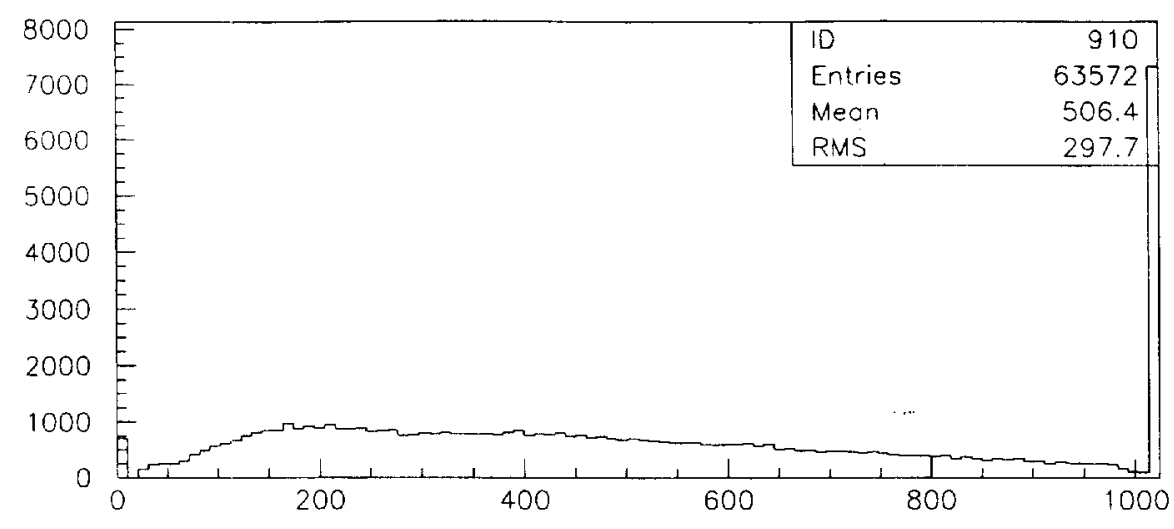

ADC distribution, electrons

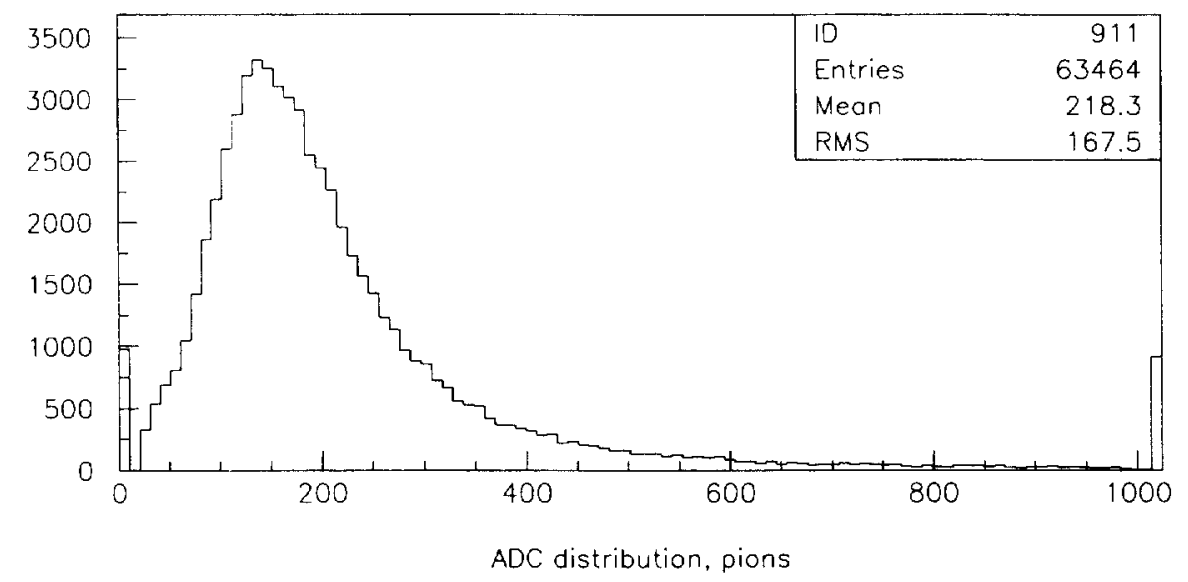

Fig. 3. Spectrum of signals from the front plane of a single TRD during the experiment: the top plot is the pulse height for electrons and the bottom that from pions.

Track and Cluster Info HCC cluster count: 0

ID Xcsi Ycsi P or E

T 1: $0.3681-0.1657-26.03$

C 2: $0.3751-0.1726 \quad 10.84$

T 2: $0.37940 .0195+13.43$

C 1: $0.3791 \quad 0.0228 \quad 13.49$

C 3: $0.5271-0.1653 \quad 0.30$

C 4: $0.2746-0.1471 \quad 1.21$

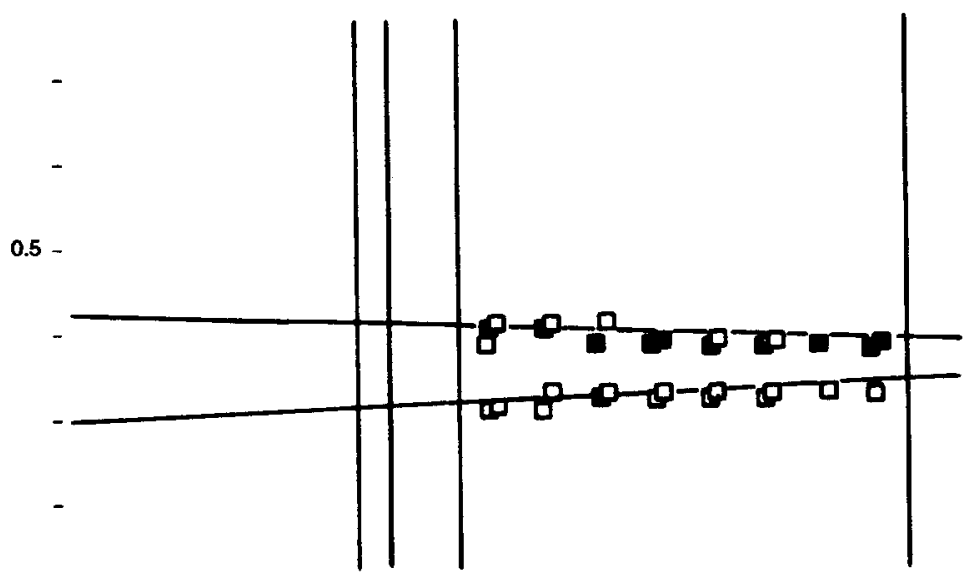

Fig. 4. Online event display during the running of the experiment showing two tracks through the TRD. One track has low ionization along the track (open squares), while the other track is mostly high ionization (filled squares), i.e. from the TR X-rays produced by an electron. 


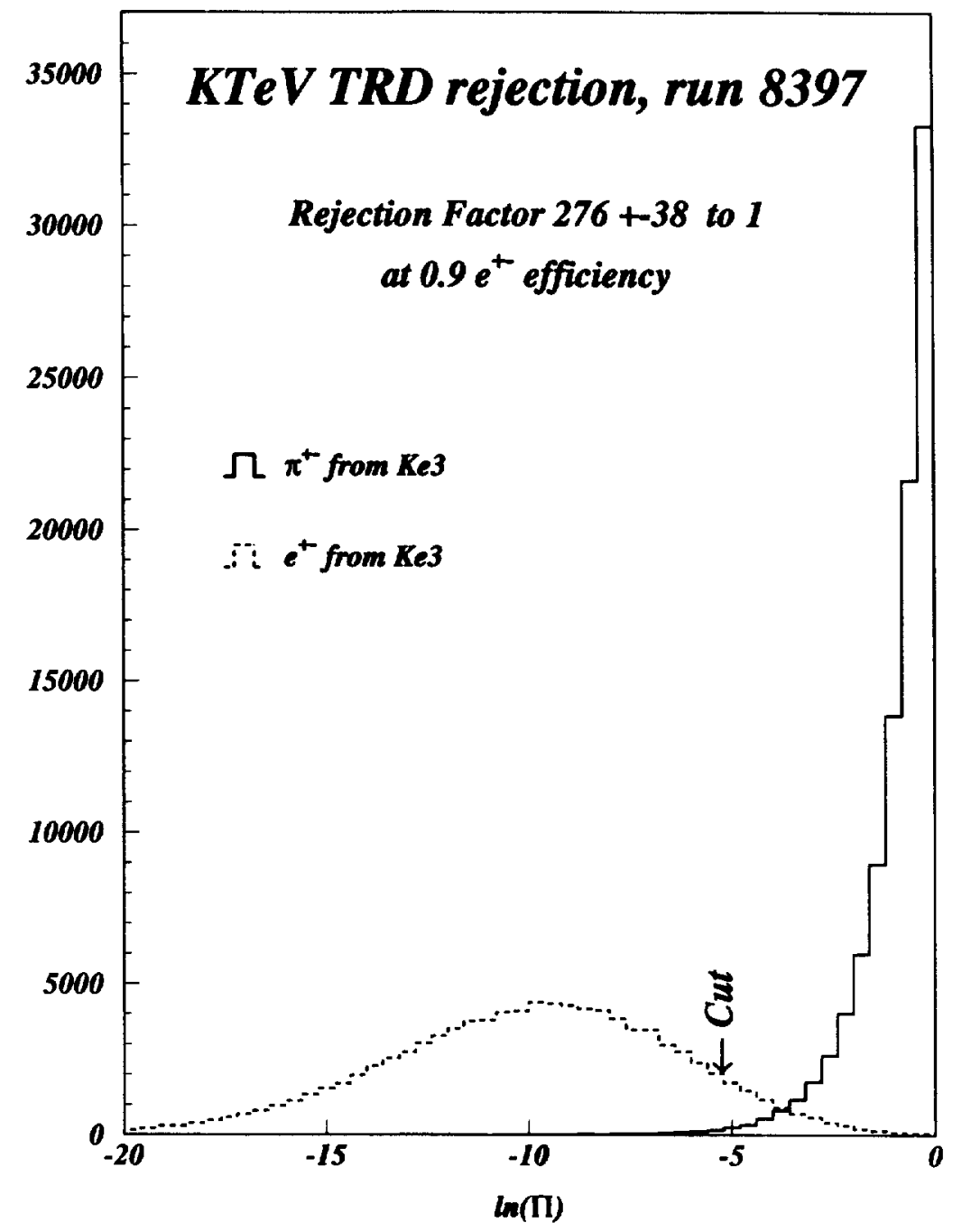

Fig. 5. The preliminary rejection from the TRD system for an early run. 